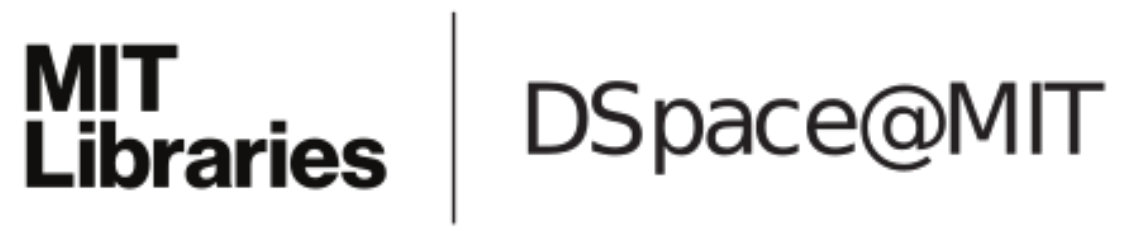

\author{
MIT Open Access Articles
}

Malleable coding with edit-distance cost

The MIT Faculty has made this article openly available. Please share how this access benefits you. Your story matters.

Citation: Varshney, L.R., J. Kusuma, and V.K. Goyal. "Malleable coding with edit-distance cost." Information Theory, 2009. ISIT 2009. IEEE International Symposium on. 2009. 204-208.๑ 2009 IEEE.

As Published: http://dx.doi.org/10.1109/ISIT.2009.5205494

Publisher: Institute of Electrical and Electronics Engineers

Persistent URL: http://hdl.handle.net/1721.1/60357

Version: Final published version: final published article, as it appeared in a journal, conference proceedings, or other formally published context

Terms of Use: Article is made available in accordance with the publisher's policy and may be subject to US copyright law. Please refer to the publisher's site for terms of use. 


\title{
Malleable Coding with Edit-Distance Cost
}

\author{
Lav R. Varshney ${ }^{1,2}$, Julius Kusuma ${ }^{3}$, and Vivek K Goyal ${ }^{1}$ \\ ${ }^{1}$ Research Laboratory of Electronics and ${ }^{2}$ Laboratory for Information and Decision Systems, MIT \\ ${ }^{3}$ Schlumberger Technology Corporation
}

\begin{abstract}
A malleable coding scheme considers not only representation length but also ease of representation update, thereby encouraging some form of recycling to convert an old codeword into a new one. We examine the trade-off between compression efficiency and malleability cost, measured with a string edit distance that introduces a metric topology to the representation domain. We characterize the achievable rates and malleability as the solution of a subgraph isomorphism problem.
\end{abstract}

\section{INTRODUCTION}

Storing information is a costly proposition. If storage is permanent, cost is determined mainly by the number of storage elements required. Therefore the length of the message representation is the key performance measure. In many storage systems, however, the message to be stored changes with time due to updates [1], [2]. Whether changing the resistance of a memristor, the molecular structure of DNA, or the inked characters on parchment, editing stored representation words is costly. Indeed there are fundamental thermodynamic costs associated with editing [3].

Unlike traditional source coding which is only concerned with the lengths of representations, malleable coding is also concerned with minimizing the cost when changing the representation to match an updated message. Denoting the original source message as $X_{1}^{n}$ and the updated source message as $Y_{1}^{n}$, suppose that a memoryless updating process $p_{Y \mid X}$ relates the two. Further denote the representation of $X_{1}^{n}$ as $A$ and the representation of $Y_{1}^{n}$ as $B$. The source distribution, the update process, and the representation mapping induce a joint distribution on the representations, $p(A, B)$, as depicted in Fig. 1. The performance metrics of interest in malleable coding are the normalized representation lengths, $\ell(A) / n$ and $\ell(B) / n$, as well as the normalized edit distance between the representations, $d(A, B) / n$, for some suitable edit distance function defined in the representation space.

Our main result for this problem is a graphical characterization of achievable rates and number of editing operations. The result involves the solution to the error-tolerant attributed subgraph isomorphism problem [4], which is essentially a graph embedding problem. Although graph functionals such as independence number and chromatic number often arise in the solution of information theory problems, this seems to be the first time that the subgraph isomorphism problem has arisen. Moreover, this is among the first treatments of a source code as a mapping between metric spaces. One might work exclusively with the Lipschitz constant of the mapping [5], but

Work supported in part by an NSF Graduate Research Fellowship and NSF Grants CCR-0325774 and CCF-0729069.

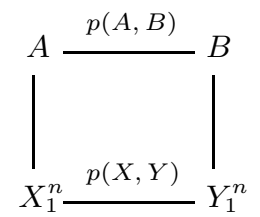

Fig. 1. Updating, representing, and editing.

our interest is in average performance rather than worst-case performance of coding schemes.

Malleable coding with edit-distance cost is described in greater detail in [6], and we refer to this easily-accessible document for proofs of our results. A distinct formulation of malleable coding is studied in [7].

\section{Problem Statement}

Consider storage medium symbols drawn from the finite alphabet $\mathcal{V}$. Unlike most source coding problems, the alphabet itself is relevant, not just the cardinality of sequences drawn from it; an abstract set of indices is not appropriate. It is natural to measure all rates in numbers of symbols from $\mathcal{V}{ }^{1}$

We require an edit distance [8] defined for $\mathcal{V}^{*}$, the set of all finite sequences of elements of $\mathcal{V}$. An example of an edit distance is the Levenshtein distance, which is constructed from insertion, deletion, and substitution operations.

Definition 1: An edit distance, $d(\cdot, \cdot)$, is a function from $\mathcal{V}^{*} \times \mathcal{V}^{*}$ to $[0, \infty)$, defined by a set of edit operations. The edit operations are a symmetric relation on $\mathcal{V}^{*} \times \mathcal{V}^{*}$. The edit distance between $a \in \mathcal{V}^{*}$ and $b \in \mathcal{V}^{*}$ is 0 if $a=b$ and is the minimum number of edit operations needed to transform $a$ into $b$ otherwise.

We define variable-length and block coding versions of our problem together, drawing distinctions only where necessary. Symbols are reused to conserve notation; context should make things clear. Let $\left\{\left(X_{i}, Y_{i}\right)\right\}_{i=1}^{\infty}$ be a sequence of independent drawings of a pair of random variables $(X, Y), X \in \mathcal{W}, Y \in$ $\mathcal{W}$, where $\mathcal{W}$ is a finite set and $p_{X, Y}(x, y)=\operatorname{Pr}[X=x, Y=$ $y]$. The joint distribution determines the marginals, $p_{X}(x)$ and $p_{Y}(y)$, as well as the modification channel, $p_{Y \mid X}(y \mid x)$. If the joint distribution is such that the marginals are equal, the modification channel is said to perform stationary updating.

Variable-length Codes: A variable-length encoder and corresponding decoder with block length $n$ are mappings $f_{E}$ : $\mathcal{W}^{n} \rightarrow \mathcal{V}^{*}$ and $f_{D}: \mathcal{V}^{*} \rightarrow \mathcal{W}^{n}$. The encoder and decoder

\footnotetext{
${ }^{1}$ This is equivalent to using base- $|\mathcal{V}|$ logarithms and all logarithms should be interpreted as such.
} 
define a variable-length code; we further require the encoderdecoder pair to be instantaneous.

A (variable-length) encoder-decoder is applied as follows. Let $(A, B)=\left(f_{E}\left(X_{1}^{n}\right), f_{E}\left(Y_{1}^{n}\right)\right)$, inducing random variables $A$ and $B$ that are drawn from the alphabet $\mathcal{V}^{*}$. Also let $\left(\hat{X}_{1}^{n}, \hat{Y}_{1}^{n}\right)=\left(f_{D}(A), f_{D}(B)\right)$.

Block Codes: A block encoder for $X$ with parameters $(n, K)$ is a mapping $f_{E}^{(X)}: \mathcal{W}^{n} \rightarrow \mathcal{V}^{n K}$, and a block encoder for $Y$ with parameters $(n, L)$ is a mapping $f_{E}^{(Y)}: \mathcal{W}^{n} \rightarrow \mathcal{V}^{n L}$. Two encoders are specified for block coding to allow different levels of compression. Given these encoders, a common decoder with parameter $n$ is $f_{D}: \mathcal{V}^{*} \rightarrow \mathcal{W}^{n}$. The encoders and decoder define a block code. Since there is a common decoder, both codes should be in the same format.

A (block) encoder-decoder with parameters $(n, K, L)$ is applied as follows. Let $(A, B)=\left(f_{E}^{(X)}\left(X_{1}^{n}\right), f_{E}^{(Y)}\left(Y_{1}^{n}\right)\right)$, inducing random variables $A \in \mathcal{V}^{n K}$ and $B \in \mathcal{V}^{n L}$. Also let $\left(\hat{X}_{1}^{n}, \hat{Y}_{1}^{n}\right)=\left(f_{D}(A), f_{D}(B)\right)$.

For both variable-length and block coding, the error rate $\Delta$ is defined as usual. Conventional performance criteria for the codes are the per-letter average lengths of codewords

$$
K=\frac{1}{n} E[\ell(A)] \text { and } L=\frac{1}{n} E[\ell(B)],
$$

where $\ell(\cdot)$ denotes the length of a sequence in $\mathcal{V}^{*}$. The final performance measure captures our novel concern with the cost of changing the coded representation. The malleability cost is the expected per-source-letter edit distance between the codes:

$$
M=\frac{1}{n} E[d(A, B)] \text {. }
$$

Definition 2: Given a source $p(X, Y)$ and an edit distance $d$, a triple $\left(K_{0}, L_{0}, M_{0}\right)$ is said to be achievable for the variable-length coding problem if, for arbitrary $\epsilon>0$, there exists (for $n$ sufficiently large) a variable-length code with error rate $\Delta=0$, average codeword lengths $K \leq K_{0}+\epsilon$, $L \leq L_{0}+\epsilon$, and malleability $M \leq M_{0}+\epsilon$.

Definition 3: Given a source $p(X, Y)$ and an edit distance $d$, a triple $\left(K_{0}, L_{0}, M_{0}\right)$ is said to be achievable for the block coding problem if, for arbitrary $\epsilon>0$, there exists (for $n$ sufficiently large) a block code with error rate $\Delta<\epsilon$, average codeword lengths $K \leq K_{0}+\epsilon, L \leq L_{0}+\epsilon$, and malleability $M \leq M_{0}+\epsilon$.

For the variable-length problem, the set of achievable ratemalleability triples is denoted $\mathfrak{P}_{V}$; for the block version, the corresponding set is denoted $\mathfrak{P}_{B}$. It follows from the definition that $\mathfrak{P}_{V}$ and $\mathfrak{P}_{B}$ are closed subsets of $\mathbb{R}^{3}$ and have the property that if $\left(K_{0}, L_{0}, M_{0}\right) \in \mathfrak{P}$, then $\left(K_{0}+\epsilon_{1}, L_{0}+\right.$ $\left.\epsilon_{2}, M_{0}+\epsilon_{3}\right) \in \mathfrak{P}$ for any $\epsilon_{i} \geq 0, i=1,2,3$. Consequently, $\mathfrak{P}_{V}$ and $\mathfrak{P}_{B}$ are completely defined by their lower boundaries, which too are closed.

Returning to Fig. 1, for given $p(X, Y)$ the malleability constraint defines what is achievable in terms of $p(A, B)$ with the additional constraints of lossless or near lossless maps between $X_{1}^{n}$ and $A$, and between $Y_{1}^{n}$ and $B$. An alternative formulation as a mapping between two metric spaces $\mathcal{W}^{n}$ and $\mathcal{V}^{*}$ is also possible.

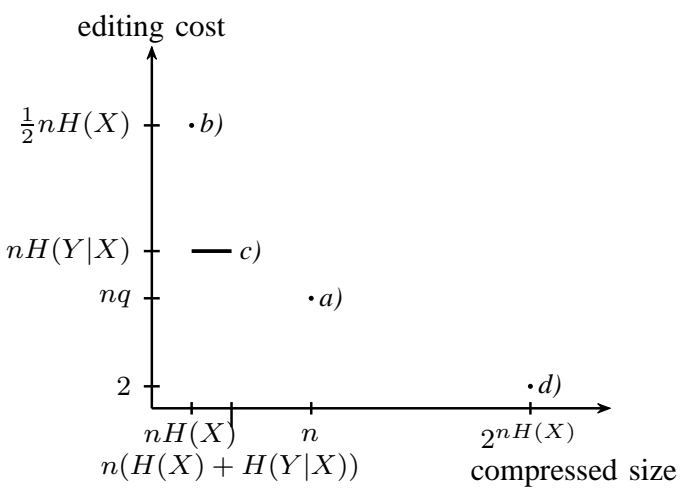

Fig. 2. Qualitative representation of the four simple techniques of Section III. For ease of representation, $H(X)=H(Y)$ is assumed. The relative orderings of points are based on $H(Y \mid X) \ll H(X)$; this reflects the natural case where the editing operation is of low complexity relative to the original string.

\section{EASILY ACHIEVED PoINTS}

To motivate the exposition, first consider four examples of how one might trade off between compression and malleability. This informal presentation is summarized in Fig. 2.

a) No compression: Taking $A=X$ and $B=Y$, it follows immediately that $K=1$ and $L=1$ and that the malleability cost is $M=E[d(X, Y)]$. If we take the edit distance to be the Hamming distance, then $M=\operatorname{Pr}[X \neq Y] \triangleq q$. Thus the triple $(K, L, M)=(1,1, q)$ is achievable.

b) Fully compress $X_{1}^{n}$ and $Y_{1}^{n}$ : One might naively apply an optimal source code. If the updating process $p_{Y \mid X}$ is stationary, then a common instantaneous code may be used to asymptotically achieve $K=H(X)$ and $L=H(Y)$; if not, then some rate loss is incurred [9]. It seems, however, that a large portion of the codeword must be changed-perhaps about half the symbols-so as to represent $Y_{1}^{n}$.

c) Fully compress $X_{1}^{n}$ and an increment: One might optimally compress the update separately and append it to the representation of $X_{1}^{n}$. The new representation has length $n(H(X)+H(Y \mid X)) \geq n H(Y)$ bits. The extended Hamming malleability cost is $n H(Y \mid X)$ symbols.

d) Completely favor malleability over compression:

Another coding scheme (due to R. G. Gallager) dramatically trades compression for malleability. The source $X_{1}^{n}$ is encoded with $2^{n H(X)}$ symbols, using an indicator function to denote which typical sequence was observed. The same strategy is used to encode $Y_{1}^{n}$, using $2^{n H(Y)}$ symbols. Updating requires substituting only two symbols when $X_{1}^{n}$ and $Y_{1}^{n}$ are different.

The coding schemes we develop will perform better than the schemes depicted in Fig. 2.

\section{CODING With GRAPH EMBEDding}

In this section, we develop a method of coding based on graph embedding and Gray codes. We then construct examples that show improved performance over naive schemes.

Before proceeding, consider some lower bounds for arbitrary sources $p(X, Y)$. From the source coding theorems, $K \geq H(X)$ and $L \geq H(Y)$. Since distinct codewords must 

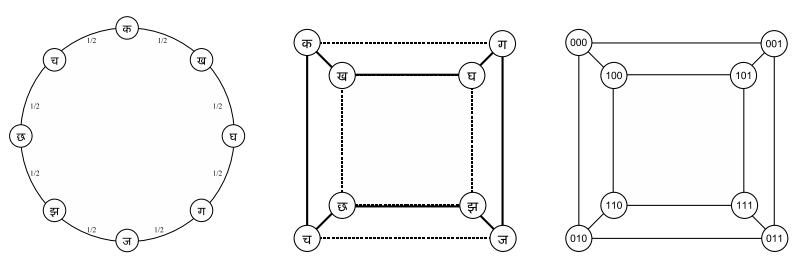

Fig. 3. (a) Weighted adjacency graph for noisy typewriter channel . (b) Graph embedded in 3-dimensional hypercube. Thick lines represent edges that are used in the embedding; dotted lines represent edges in the hypercube that are unused in the embedding. (c) Hypercube graph labeled with binary reflected Gray code.

have an edit distance of at least one, we can lower bound $M$ by assuming that minimal distance. Then edit distance is simply the probability of error for uncoded transmission. For $n=1, M \geq \sum_{x \in \mathcal{W}} \sum_{y \in \mathcal{W}: y \neq x} p(x, y)$ and more generally,

$$
M \geq \frac{1}{n} \sum_{x_{1}^{n} \in \mathcal{W}^{n}} \sum_{y_{1}^{n} \in \mathcal{W}^{n}: y_{1}^{n} \neq x_{1}^{n}} p\left(x_{1}^{n}, y_{1}^{n}\right) .
$$

A weaker, simplified version of the bound is $M \geq \frac{1}{n}$, which is a worst-case measure.

Now we construct an example that simultaneously achieves the rate lower bounds and the malleability lower bound (1). Consider a memoryless, equiprobable source $p(x)$ with alphabet $\mathcal{W}=\{$ क, ख, घ, ग, ज, झ, छ, च\}, and thus $H(X)=3$ bits. Consider the noisy typewriter update process where the probability of making an error in either direction is $1 / 4$. Evidently, the bound on $M$ is $1 / 2$ for $n=1$. Moreover, the $Y$ marginal is also equiprobable, with $L$ bounded by 3 bits.

Take $\mathcal{V}$ to be $\{0,1\}$ and develop a binary encoding scheme using graph embedding methods. Draw a graph where the vertices are the symbols and the edges are labeled with total transition probabilities. The result is a weighted adjacency graph, a weighted version of the adjacency graphs in [10], [11], as shown in Fig. 3(a).

Suppose that the edit distance is the Hamming distance. Now try to embed this adjacency graph into a hypercube of a given size, first considering size 3 . The adjacency graph is exactly embeddable into the hypercube, as shown in Fig. 3(b). If it were not exactly embeddable, some of the low weight edges might have to be broken. After embedding into the hypercube, use the binary reflected Gray code (see [12] and Fig. 3(c) for a description) to assign codewords through correspondence.

Clearly the code is lossless so the error rate is $\Delta=0$. Since all codewords are of length 3 , clearly $K=L=3$. To compute $M$, notice that any source symbol is perturbed to any one of its neighbors with probability $1 / 2$. Further notice that the Hamming distance between neighbors in the hypercube is 1 . Thus $M=1 / 2$. This encoding scheme achieves the entropy bounds $H(X)$ and $H(Y)$. It also achieves the $n=1$ lower bound for $M$ and is thus optimal for $n=1$.

Since the embedding relation is true for $n=1$, it is also true that $n$-fold Cartesian products of the adjacency graph are embeddable into $n$-fold Cartesian products of the hypercube. Such a scheme would achieve rates of $K=3$ bits and $L=3$ bits. It would also achieve $M$ of $\frac{1}{n} \operatorname{Pr}\left[X_{1}^{n} \neq Y_{1}^{n}\right]$ since the Cartesian product of the adjacency graph exactly represents edit costs of 1 . For each $n$, this matches the lower bound (1), and is thus optimal. Furthermore, asymptotically in $n$, the triple $(K, L, M)=(3,3,0)$ is achievable.

Observe that embeddability into a graph where graph distance corresponds to edit distance seems to be sufficient to guarantee good performance; we will explore this in detail in the sequel.

Similar constructions are possible for variable-length codes. When using such codes, the appropriate edit distance might be the Levenshtein distance, so a minimal change codelabeled Levenshtein distance graph rather than a Gray codelabeled hypercube would be used. When embedding in other graphs, codeword lengths must also be taken into account. If a common Huffman code for $p_{X}$ and for $p_{Y}$ is embeddable (with matched vertex labels) in the Levenshtein graph, then minimal $K, L$, and $M$ are simultaneously achievable.

\section{General Characterizations}

Using the insights garnered from the example, detailed characterizations of the set of achievable rate-malleability triples are obtained. For variable-length coding, results are expressed in terms of the solution to an error-tolerant attributed subgraph isomorphism problem [4].

\section{A. Error-Tolerant Attributed Subgraph Isomorphism}

A vertex-attributed graph is a three-tuple $G=(V, E, \mu)$, where $V$ is the set of vertices, $E \subseteq V \times V$ is the set of edges, and $\mu: V \rightarrow \mathcal{V}^{*}$ is a function assigning labels to vertices. The set of labels is denoted $\mathcal{V}^{*}$.

Definition 4: Consider two vertex-attributed graphs $G=$ $\left(V(G), E(G), \mu_{G}\right)$ and $H=\left(V(H), E(H), \mu_{H}\right)$. Then $G$ is said to be embeddable into $H$ if $H$ has a subgraph isomorphic to $G$. That is, there is an injective map $\phi: V(G) \rightarrow V(H)$ such that $\mu_{G}(v)=\mu_{H}(\phi(v))$ for all $v \in V(G)$ and that $(u, v) \in E(G)$ implies $(\phi(u), \phi(v)) \in E(H)$. This is denoted as $G \rightsquigarrow H$.

Several graph editing operations may be defined, such as substituting a vertex label, deleting a vertex, deleting an edge, and inserting an edge. An edited graph is denoted through the operator $\mathcal{E}(\cdot)$ corresponding to the sequence of graph edit operations $\mathcal{E}=\left(e_{1}, \ldots, e_{k}\right)$. There is a cost associated with each sequence of graph edit operations.

Definition 5: Given two graphs $G$ and $H$, an error-tolerant attributed subgraph isomorphism $\psi$ from $G$ to $H$ is the composition of two operations $\psi=\left(\mathcal{E}, \phi_{\mathcal{E}}\right)$ where

- $\mathcal{E}$ is a sequence of graph edit operations such that there exists an $\mathcal{E}(G)$ that satisfies $\mathcal{E}(G) \rightsquigarrow H$.

- $\phi_{\mathcal{E}}$ is an embedding of $\mathcal{E}(G)$ into $H$.

Definition 6: The subgraph distance $\rho(G, H)$ is the cost of the minimum cost error-correcting attributed subgraph isomorphism $\psi$ from $G$ to $H$.

Note that in general, $\rho(G, H) \neq \rho(H, G)$. 


\section{B. Closeness Vitality}

The subgraph isomorphism cost structure for malleable coding is based on a graph theoretic quantity closeness vitality [13]. An edge vitality index is the difference between some functional of a graph and that same functional of the graph with an edge removed.

Let $f_{W}(G)$ of a graph $G$ be the sum of the distances of all vertex pairs:

$$
f_{W}(G)=\sum_{v \in V} \sum_{w \in V} d(v, w) .
$$

Definition 7: The closeness vitality $\operatorname{cv}(G, r)$ of graph $G$ with respect to edge $r$ is: $\operatorname{cv}(G, r)=f_{W}(G)-f_{W}(G / r)$.

\section{C. $\mathfrak{P}_{V}$ Characterization}

We are concerned with the error-tolerant embedding of an attributed, weighted source adjacency graph into the graph induced by a $\mathcal{V}^{*}$-space edit distance. Edge deletion is the only graph editing operation that we need.

First consider the delay-free case, $n=1$. A source $p(X, Y)$ and an edit distance $d(\cdot, \cdot)$ are given. Huffman coding provides the minimal redundancy instantaneous code and achieves expected performance $H(X) \leq K \leq H(X)+1$. Similarly, a Huffman code for $Y$ yields $H(Y) \leq L \leq H(Y)+1$. The rate loss for using an incorrect Huffman code is essentially a divergence quantity [9]. A source code may be thought of in terms of a random variable, here $Z$. For a given $Z$, there are several Huffman codes: those arising from different labelings of the code tree and also perhaps different trees [14]. Let us denote the set of all Huffman codes for $Z$ as $\mathcal{H}_{Z}$.

Since $K$ and $L$ are fixed by the choice of $Z$, all that remains is to determine the set of achievable $M$. Let $G$ be the graph induced by the edit distance $d(\cdot, \cdot)$, and $d_{G}$ its path metric. The graph $G$ is intrinsically labeled. Let $A$ be the weighted adjacency graph of the source $p(X, Y)$, with vertices $\mathcal{W}$, edges $E(A) \subseteq \mathcal{W} \times \mathcal{W}$, and labels given by a Huffman code. That is, $A=\left(\mathcal{W}, E(A), f_{E}\right)$ for some $f_{E} \in \mathcal{H}_{Z}$. There is a path semimetric, $d_{A}$, associated with the graph $A$.

The basic problem is to solve the error-tolerant subgraph isomorphism problem of embedding $A$ into $G$. In general for $n=1$, the malleability cost under edit distance $d_{G}$ when using the source code $f_{E}$ is

$$
M=\sum_{x \in \mathcal{W}} \sum_{y \in \mathcal{W}} p(x, y) d_{G}\left(f_{E}(x), f_{E}(y)\right) .
$$

The smallest malleability possible is when $A$ is a subgraph of $G$, and then

$$
\begin{aligned}
M_{\min } & =\sum_{x \in \mathcal{W}} \sum_{y \in \mathcal{W}} p(x, y) d_{A}(x, y) \\
& =\sum_{x \in \mathcal{W}} \sum_{y \in \mathcal{W}} p(x, y) d_{G}\left(f_{E}(x), f_{E}(y)\right) \\
& =E\left[f_{W}(A)\right]=\operatorname{Pr}[X \neq Y] .
\end{aligned}
$$

If edges in $A$ need to broken for embedding, $M$ increases. If an edge $\bar{e}$ is removed from the graph $A$, the resulting graph $A / \bar{e}$ induces its own path semimetric $d_{A / \bar{e}}$. The cost of removing edge $\bar{e}$ from the graph $A$ is:

$$
\sum_{x, y \in \mathcal{W}} p(x, y)\left[d_{A / \bar{e}}\left(f_{E}(x), f_{E}(y)\right)-d_{A}\left(f_{E}(x), f_{E}(y)\right)\right],
$$

which is the following function of the associated removal operation $e$ :

$$
C(e)=-E[\operatorname{cv}(A, \bar{e})] .
$$

If $\mathcal{E}$ is a sequence of edge removals, $\overline{\mathcal{E}}$, then $C(\mathcal{E})=$ $-E[\operatorname{cv}(A, \overline{\mathcal{E}})]$. Putting things together, $\mathfrak{P}_{V}$ contains any point

$$
\begin{aligned}
K & =H(X)+D\left(p_{X} \| p_{Z}\right)+1, \\
L & =H(Y)+D\left(p_{Y} \| p_{Z}\right)+1, \\
M & =M_{\min }+\min _{f_{E} \in \mathcal{H}_{Z}} \rho(A, G) .
\end{aligned}
$$

Increasing the block length beyond $n=1$ may improve performance, which we show in the following.

Theorem 1: Consider a source $p(X, Y)$ with associated (unlabeled) weighted adjacency graph $A$ and an edit distance $d$ with associated graph $G$. For any $n$, let $\mathfrak{P}_{V}^{(a c h)}$ be the set of triples $(K, L, M)$ that are computed, by allowing an arbitrary choice of the memoryless random variable $p\left(Z_{1}^{n}\right)$, as follows:

$$
\begin{aligned}
K & =H(X)+D\left(p_{X} \| p_{Z}\right)+\frac{1}{n}, \\
L & =H(Y)+D\left(p_{Y} \| p_{Z}\right)+\frac{1}{n}, \\
M & =\frac{1}{n} \operatorname{Pr}\left[X_{1}^{n} \neq Y_{1}^{n}\right]+\frac{1}{n} \min _{f_{E} \in \mathcal{H}_{Z_{1}^{n}}^{n}} \rho\left(\left(\mathcal{W}^{n}, E(A), f_{E}\right), G\right) .
\end{aligned}
$$

Then the set of triples $\mathfrak{P}_{V}^{(a c h)}$ is achievable instantaneously.

The theorem, proven in [6], states that error-tolerant subgraph isomorphism implies achievable malleability. The choice of the auxiliary random variable $Z$ is open to optimization. If minimal rates are desired, $p_{Z}$ must be on the geodesic connecting $p_{X}$ and $p_{Y}$. If $Z$ is not on the geodesic, then there is some rate loss, but perhaps also some malleability gains.

When $p_{Y \mid X}$ is a stationary update process, the simple lower bounds might be tight to this achievable region.

Corollary 1: Consider a source as given above in Theorem 1. If $p_{Y \mid X}$ is stationary, $p_{X}=p_{Y}$ is $|\mathcal{V}|$-adic, and there is a Huffman-labeled $A$ for $p_{X}=p_{Y}$ that is an isometric subgraph of $G$, then the block length $n$ lower bound $\left(H(X), H(Y), \frac{1}{n} \operatorname{Pr}\left[X_{1}^{n} \neq Y_{1}^{n}\right]\right)$ is tight to this achievable region for every $n$, and in particular to $(H(X), H(Y), 0)$ for large $n$.

\section{D. $\mathfrak{P}_{B}$ Characterization}

Now we turn our attention to the block-coding problem. For $\mathfrak{P}_{B}$, we use a joint typicality graph rather than the weighted adjacency graph used for $\mathfrak{P}_{V}$. Additionally we focus on binary block codes under Hamming edit distance, so we are concerned only with hypercubes rather than general edit distance graphs. We use standard typicality notations, definitions, and arguments from [15].

For the bivariate distribution $p_{X, Y}$, define a square matrix called the strong joint typicality matrix $A_{[X Y]}^{n}$ as follows. There is one row (and column) for each sequence in $S_{[X] \delta}^{n} \cup$ 
$S_{[Y] \delta}^{n}$. The entry with row corresponding to $x_{1}^{n}$ and column corresponding to $y_{1}^{n}$ receives a one if $\left(x_{1}^{n}, y_{1}^{n}\right)$ is strongly jointly typical and zero otherwise.

Let us temporarily restrict to stationary update: $\mathcal{P}=$ $\{p(x, y) \mid p(x)=p(y)\}$. Asymptotically, $A_{[X Y]}^{n}$ will have approximately equal numbers of ones in all columns and in all rows. Think of $A_{[X Y]}^{n}$ as the adjacency matrix of a graph, where the vertices are sequences and edges connect sequences that are jointly typical with one another.

Proposition 1: Take $A_{[X Y]}^{n}$ for some source in $\mathcal{P}$ as the adjacency matrix of a graph $\mathcal{G}^{n}$. The number of vertices in the graph will satisfy

$$
(1-\delta) 2^{n(H(X)-\psi)} \leq\left|V\left(\mathcal{G}^{n}\right)\right| \leq 2^{n(H(X)+\psi)},
$$

where $\psi \rightarrow 0$ as $n \rightarrow \infty$ and $\delta \rightarrow 0$. The degree of each vertex, $\operatorname{deg}_{v}$, will concentrate as

$$
2^{n(H(Y \mid X)-\nu)} \leq \operatorname{deg}_{v} \leq 2^{n(H(Y \mid X)+\nu)},
$$

where $\nu \rightarrow 0$ as $n \rightarrow \infty$ and $\delta \rightarrow 0$.

The basic topology of the strongly typical set is asymptotically a $2^{n H(Y \mid X)}$-regular graph on $2^{n H(X)}$ vertices. Graph embedding ideas then yield a theorem on block coding achievability:

Theorem 2: For a source $p(x, y) \in \mathcal{P}$ and the Hamming edit distance, a triple $\left(K, K, M=M_{\min }\right)$ is achievable if $\mathcal{G}^{n} \rightsquigarrow H_{n K}$, where $H_{n K}$ is the hypercube of size $n K$.

Using this result, we argue that a linear increase in malleability is at exponential cost in code length. A simple counting argument leads to a condition for embeddability.

Theorem 3: For a source $p(x, y) \in \mathcal{P}$, if asymptotically $\mathcal{G}^{n} \rightsquigarrow H_{n K}$ then

$$
n K \geq \max \left(n H(X), 2^{n H(Y \mid X)}\right) .
$$

The space $S^{n} \triangleq S_{[X] \delta}^{n} \cup S_{[Y] \delta}^{n}$ with the corresponding path metric, $d_{A}$ induced by $A_{[X Y]}^{n}$ is a metric space. Hypercubes with their natural path metric, $d_{G}$, are also metric spaces. Rather than requiring absolutely minimal $n M$, it can be noted that $M$ is asymptotically zero when the Lipschitz constant associated with the mapping between the source space and the representation space has nice properties in $n$.

Definition 8: A mapping between metric spaces $f$ : $\left(S^{n}, d_{A^{n}}\right) \rightarrow\left(\mathcal{V}^{n K}, d_{G^{n}}\right)$ is called Lipschitz continuous if

$$
d_{G^{n}}\left(f\left(x_{1}\right), f\left(x_{2}\right)\right) \leq C d_{A^{n}}\left(x_{1}, x_{2}\right)
$$

for some constant $C$ and for all $x_{1}, x_{2} \in S^{n}$. The smallest such $C$ is the Lipschitz constant, $\operatorname{Lip}[f]$.

We can bound the malleability of a coding scheme that only represents sequences in $S^{n}$ in terms of the Lipschitz constant.

Theorem 4: For a coding scheme $f_{E}$ that only represents sequences in $S^{n}=S_{[X] \delta}^{n} \cup S_{[Y] \delta}^{n}$,

$$
M \leq \frac{\operatorname{Lip}\left[f_{E}\right]}{n}\left(1+\delta \operatorname{diam}\left(\mathcal{G}^{n}\right)\right),
$$

where $\operatorname{diam}(\cdot)$ is the graph diameter.

Results from theoretical computer science [6] and some source coding constructions [5] may provide further characterization of $\operatorname{Lip}\left[f_{E}\right]$.

\section{Discussion AND CONCLUSIONS}

We have formulated information theoretic problems motivated by costly writing on storage media. The problems exhibit a trade-off between compression efficiency and the costs incurred when updating using random access editing.

For the zero-error problem, we found that the subgraph distance between a source graph and a storage medium graph determines the rate-malleability relation. Since index assignment for joint source channel coding, signal constellation labeling, and this problem are similar, it is not surprising that Gray codes arise in each [12], [16]. All involve a transformation of objects of one kind into objects of a new kind so that the distances in the two spaces are approximately equal [8].

For block coding, we found that if minimal malleability costs are desired, then a rate penalty that is exponential in the conditional entropy of the update process must be paid. That is, unless the two versions of the source are very strongly correlated (conditional entropy logarithmic in block length), rate exponentially larger than entropy is needed. If we require malleability $M=O(1 / n)$, then rates $K$ and $L$ must be $\Omega\left(\frac{1}{n} 2^{n}\right)$.

\section{ACKNOWLEDGMENTS}

Discussions with V. Tarokh, R. G. Gallager, S. K. Mitter, S. Tatikonda, and R. K. Sastry are appreciated.

\section{REFERENCES}

[1] D. R. Bobbarjung, S. Jagannathan, and C. Dubnicki, "Improving duplicate elimination in storage systems," ACM Trans. Storage, vol. 2, no. 4 pp. 424-448, Nov. 2006.

[2] R. Burns, L. Stockmeyer, and D. D. E. Long, "In-place reconstruction of version differences," IEEE Trans. Knowl. Data Eng., vol. 15, no. 4, pp. 973-984, July-Aug. 2003.

[3] C. H. Bennett, et al., "Information distance," IEEE Trans. Inf. Theory, vol. 44, no. 4, pp. 1407-1423, July 1998.

[4] B. T. Messmer and H. Bunke, "A new algorithm for error-tolerant subgraph isomorphism detection," IEEE Trans. Pattern Anal. Mach. Intell., vol. 20, no. 5, pp. 493-504, May 1998.

[5] A. Montanari and E. Mossel, "Smooth compression, Gallager bound and nonlinear sparse-graph codes," in Proc. 2008 IEEE Int. Symp. Inf. Theory, July 2008, pp. 2474-2478.

[6] L. R. Varshney, J. Kusuma, and V. K. Goyal, "Malleable coding: Compressed palimpsests," arXiv:0806.4722v1 [cs.IT]., June 2008.

[7] J. Kusuma, L. R. Varshney, and V. K. Goyal, "Malleable coding with fixed segment reuse," arXiv:0809.0737v1 [cs.IT]., Sept. 2008.

[8] G. Cormode, "Sequence distance embeddings," Ph.D. dissertation, University of Warwick, Warwick, Jan. 2003.

[9] E. N. Gilbert, "Codes based on inaccurate source probabilities," IEEE Trans. Inf. Theory, vol. IT-17, no. 3, pp. 304-314, May 1971.

[10] C. E. Shannon, "The zero error capacity of a noisy channel," IRE Trans. Inf. Theory, vol. IT-2, no. 3, pp. 8-19, Sept. 1956.

[11] H. S. Witsenhausen, "The zero-error side information problem and chromatic numbers," IEEE Trans. Inf. Theory, vol. IT-22, no. 5, pp. 592-593, Sept. 1976.

[12] E. Agrell, J. Lassing, E. G. Ström, and T. Ottosson, "On the optimality of the binary reflected Gray code," IEEE Trans. Inf. Theory, vol. 50, no. 12 , pp. 3170-3182, Dec. 2004.

[13] D. Koschützki, et al., "Centrality indices," in Network Analysis: Methodological Foundations, Berlin: Springer, 2005, pp. 16-61.

[14] R. Ahlswede, "Identification entropy," in General Theory of Information Transfer and Combinatorics, Berlin: Springer, 2006, pp. 595-613.

[15] R. W. Yeung, A First Course in Information Theory. New York: Kluwer Academic/Plenum Publishers, 2002.

[16] K. Zeger and A. Gersho, "Pseudo-Gray coding," IEEE Trans. Commun., vol. 38, no. 12, pp. 2147-2158, Dec. 1990. 